\title{
12(S)-HETE mediates diabetes-induced endothelial dysfunction by activating intracellular endothelial cell TRPV1
}

\author{
Mandy Otto, ${ }^{1}$ Clarissa Bucher, ${ }^{1}$ Wantao Liu, ${ }^{1}$ Melanie Müller, ${ }^{1}$ Tobias Schmidt,,${ }^{1,2}$ Marina Kardell, ${ }^{1}$ Marvin Noel Driessen, ${ }^{1}$ \\ Jan Rossaint, ${ }^{1}$ Eric R. Gross, ${ }^{3}$ and Nana-Maria Wagner ${ }^{1}$ \\ 1Department of Anesthesiology, Intensive Care and Pain Medicine, and ${ }^{2}$ Institute of Physiology I, University Hospital Münster, Münster, Germany. ${ }^{3}$ Department of Anesthesiology, \\ Perioperative and Pain Medicine, Stanford University, Stanford, California, USA.
}

\begin{abstract}
Patients with diabetes develop endothelial dysfunction shortly after diabetes onset that progresses to vascular disease underlying the majority of diabetes-associated comorbidities. Increased lipid peroxidation, mitochondrial calcium overload, and mitochondrial dysfunction are characteristics of dysfunctional endothelial cells in diabetic patients. We here identified that targeting the lipid peroxidation product 12(S)-hydroxyeicosatetraenoic acid-induced [12(S)-HETE-induced] activation of the intracellularly located cation channel transient receptor potential vanilloid 1 (TRPV1) in endothelial cells is a means to causally control early-stage vascular disease in type I diabetic mice. Mice with an inducible, endothelium-specific 12/15-lipoxygenase (12/15Lo) knockout were protected similarly to TRPV1-knockout mice from type 1 diabetes-induced endothelial dysfunction and impaired vascular regeneration following arterial injury. Both 12(S)-HETE in concentrations found in diabetic patients and TRPV1 agonists triggered mitochondrial calcium influx and mitochondrial dysfunction in endothelial cells, and 12(S)-HETE effects were absent in endothelial cells from TRPV1-knockout mice. As a therapeutic consequence, we found that a peptide targeting 12(S)-HETE-induced TRPV1 interaction at the TRPV1 TRP box ameliorated diabetes-induced endothelial dysfunction and augmented vascular regeneration in diabetic mice. Our findings suggest that pharmacological targeting of increased endothelial lipid peroxidation can attenuate diabetes-induced comorbidities related to vascular disease.
\end{abstract}

\section{Introduction}

Millions of people worldwide suffer from diabetes, and the numbers are increasing. Among children, the incidence of type 1 diabetes mellitus currently doubles every 20 years (1). Diabetes leads to multiple comorbidities, such as heart disease, kidney dysfunction, loss of vision, and impaired wound healing, that reduce quality of life and life expectancy of diabetic patients (2). These comorbidities are almost exclusively attributable to vascular disease that is initiated by diabetes-induced loss of endothelial integrity. In children who - apart from hyperglycemia - do not exhibit any additional cardiovascular risk factors, the endothelium loses its ability to regulate vasotonus already a few years after disease onset (3). Endothelial dysfunction correlates to vascular disease progression and severity that are relevant to the outcome of patients with diabetes (4). Despite guideline-based medical therapy, diabetic patients develop comorbidities secondary to vascular disease with an unacceptably high incidence. Moreover, the mechanisms of diabetes-induced vascular pathology are incompletely under-

Conflict of interest: ERG holds a patent on "Peptide modulators of specific calcineurin protein-protein interactions" (US Application Serial No. 16/082,313) that includes the V1-cal peptide.

Copyright: (5) 2020, American Society for Clinical Investigation.

Submitted: January 21, 2020; Accepted: June 17, 2020; Published: August 17, 2020

Reference information: J Clin Invest. 2020;130(9):4999-5010.

https://doi.org/10.1172/JCl136621. stood, and therapeutic options specifically targeting vascular disease initiation in diabetes are lacking.

In patients with diabetes, endothelial cells exhibit damaged mitochondria, mitochondrial dysfunction, and a decreased oxidative capacity (5). Increasing evidence identifies that mitochondrial dysfunction is linked to mitochondrial calcium overload that contributes to loss of mitochondrial membrane potential, impaired mitochondrial respiration, and excessive generation of mitochondrial reactive oxygen species (6). The defects in mitochondrial calcium handling further correlate with a reduced endothelial bioavailability of nitric oxide that results in defective endothelium-dependent vasodilation, one of the central characteristics of diabetes-induced endothelial dysfunction $(7,8)$. However, the mediators of diabetes-induced disturbances of mitochondrial calcium homeostasis have not been fully elucidated so far, and there is currently no option to causally control early-stage vascular disease in diabetes.

Hyperglycemia induces increased arachidonic acid breakdown by 12-lipoxygenases (12LOXs) in endothelial cells that results in increased production of endothelial 12(S)-hydroperoxy-5Z,8Z,10E,14Z-eicosatetraenoic acid [12(S)-HpETE] and its stable metabolite 12(S)-hydroxyeicosatetraenoic acid [12(S)-HETE] (9). 12(S)-HETE plasma concentrations are particularly elevated in children with newly diagnosed type 1 diabetes (10) and adult diabetic patients with vascular complications such as coronary artery disease and early renal disease $(11,12)$. However, it is unclear to 
what extent and how 12(S)-HETE contributes to vascular pathology in diabetes. 12(S)-HETE is a potent activator of transient receptor potential vanilloid 1 (TRPV1) (13). TRPV1 is a nonselective cation channel primarily gating calcium that is activated by physical and chemical stimuli, such as heat, acidic conditions, and capsaicin, the pungent compound of the red chili pepper (14). In addition to its expression in sensory neurons, where it transmits the sensation of pain in response to noxious stimuli, TRPV1 is expressed in vascular cells, including endothelial cells (15). While TRPV1 has been intensively characterized at the cell surface, recent studies suggest intracellular localizations of TRPV1 and that TRPV1 plays a fundamental role in cellular calcium homeostasis (16). TRPV1 is implicated in the constitutive regulation of mitochondrial calcium influx (17), and mitochondrial calcium changes induced by TRPV1 activation result in mitochondrial dysfunction and can trigger calciumdependent cellular dysfunction (18).

We here aimed to identify a previously unrecognized mechanism of 12(S)-HETE-induced TRPV1 activation that crucially mediates early-stage vascular disease initiation in a mouse model of type 1 diabetes. By generating endothelium-specific 12/15-lipoxygenase-knockout (12/15LO-knockout) mice and using TRPV1-knockout mice, we showed that both 12(S)-HETE and TRPV1 are required for the development of endothelial dysfunction in diabetic mice. In vitro we verified that, by activating intracellular TRPV1 in endothelial cells, 12(S)-HETE induces mitochondrial calcium changes that result in mitochondrial dysfunction. As a therapeutic implication of this mechanism, we show that a peptide constituting an amino acid sequence of the highly conserved TRP box at the C-terminal end of TRPV1 identified as a potential 12(S)-HETE/TRPV1 interaction site can serve as a decoy and ameliorate TRPV1 activation in the presence of 12(S)-HETE, thus protecting mice from diabetesinduced endothelial dysfunction.

\section{Results}

12(S)-HETE is a mediator of diabetes-induced endothelial dysfunction and mitochondrial dysfunction in endothelial cells. To assess a role of endothelium-derived 12(S)-HETE in diabetes-induced endothelial dysfunction, we generated mice with an endothelium-specific, tamoxifen-inducible knockout of the 12(S)-HETE-generating enzyme 12/15-lipoxygenase (12/15LO in mice) by breeding homozygous $12 / 15 \mathrm{Lo} \mathrm{o}^{\mathrm{fl} / \mathrm{fl}}$ mice exhibiting loxP sites flanking exons $2-5$ of the $12 / 15$ Lo gene (19) to homozygosity with mice expressing Cre recombinase under the tamoxifen-inducible endothelium-specific vascular endothelial cadherin (VE-cadherin; Cdh5) promoter (ref. 20 and Figure 1A). Type 1 diabetes was induced in adult mice by a single streptozotocin injection, and similarly increased blood glucose levels were verified in WT, tamoxifen-treated Cre-negative, and Cre-positive mice 4 days and 4 weeks after injection (Supplemental Figure 1; supplemental material available online with this article; https://doi.org/10.1172/JCI136621DS1). While Cre-negative diabetic mice showed an increase in plasma 12(S)-HETE levels under diabetic conditions that was comparable to the increase seen in diabetic WT mice $(361.3 \pm 49.1$ and $499.8 \pm 137.3 \mathrm{ng} / \mathrm{mL}$ vs. 92.5 \pm 15.5 and $155.2 \pm 67.3 \mathrm{ng} / \mathrm{mL}$ in nondiabetic respective controls), Cre-positive diabetic mice exhibited no increase in 12(S)-HETE plasma levels in the presence of type 1 diabetes $(215.5 \pm 51.4$ vs. $177 \pm 33.4 \mathrm{ng} / \mathrm{mL}$ in nondiabetic Cre-positive control mice) (Sup- plemental Figure 2). Four weeks after streptozotocin injection, impaired endothelium-dependent vasodilation was exhibited in diabetic, tamoxifen-treated $C r e$-negative control mice $(P<0.001$ vs. nondiabetic tamoxifen-treated Cre-negative mice) that was partly restored in endothelium-specific $12 / 15 \mathrm{Lo}$-knockout mice $(P<0.001$ vs. diabetic Cre-negative animals; Figure 1, B and C). In a second model, we compared the diabetic vascular response to injury by morphometrically assessing neointimal hyperplasia 3 weeks after ferric chloride-induced carotid artery injury. While diabetic Cre-negative mice showed increased neointima area and luminal stenosis compared with nondiabetic control mice $(P$ $<0.001$ and $P<0.05$, respectively), Cre-positive diabetic mice exhibited similarly reduced neointima area and luminal stenosis compared with nondiabetic mice (Supplemental Figure 3). We next assessed whether exogenous administration of 12(S)-HpETE, the primary 12LOX metabolite and precursor of 12(S)-HETE, to human endothelial cells in vitro can affect mitochondrial integrity and endothelial cell function under normal-glucose conditions. Exposure of endothelial cells to 12(S)-HpETE in concentrations reported in patients with diabetes induced mitochondrial calcium influx and reduced mitochondrial membrane potential. Similar concentrations of 12(S)-HpETE impaired mitochondrial respiration (Figure 1, D-F) and reduced the ability of endothelial cells to form capillary-like networks on extracellular matrix (Supplemental Figure 4). To test whether increased 12(S)-HpETE levels contribute to impaired mitochondrial function induced by high levels of glucose, effects of 12LOX inhibitors were evaluated. Culture of endothelial cells under high-glucose conditions (30 mM) showed impaired mitochondrial function that was restored by exposure to the 12LOX inhibitors baicalein and phenidone (Figure 1, G and H).

Expression and function of TRPV1 in human endothelial cells. 12(S)-HpETE is a potent agonist of TRPV1, inducing calcium flux (13). Exposure of patched endothelial cells to $10 \mu \mathrm{M}$ capsaicin resulted in robust currents that were antagonized with $10 \mu \mathrm{M}$ of the specific TRPV1 antagonist 4-(3-chloro-2-pyridinyl)- $N$-[4-(1,1dimethylethyl)phenyl]-1-piperazine-carboxamide (BCTC) (Figure 2A). Western blot using TRPV1-specific antibodies (21) revealed the expression of an approximately $95-\mathrm{kDa}-$ molecular weight protein. At the cell surface, TRPV1 is found glycosylated, resulting in a molecular weight of about $110 \mathrm{kDa}(22)$, the protein size detected in murine dorsal root ganglion cells particularly enriched in TRPV1. In accordance with previous observations in other cell types such as cardiomyocytes (23), the existence of the $95 \mathrm{kDa}$ protein size suggests that TRPV1 is located not only at the plasma membrane on endothelial cells but also intracellularly. Immunohistochemical analysis revealed that TRPV1 colocalizes with endothelial cell mitochondria (Figure 2C), and cell fractionation showed TRPV1 particularly enriched in organelle fractions containing mitochondria (Figure 2B). Further functional analysis of TRPV1 using capsaicin as agonist revealed an increase in cytosolic calcium in endothelial cells upon exposure that persisted when calcium was removed from extracellular medium (Figure 2D). Similar increases in calcium levels were observed in endothelial cell mitochondria, but these effects were absent after depletion of intracellular calcium stores (Figure 2E), suggesting that TRPV1 mobilizes and gates calcium from intracellular stores into the cytosol and - primarily or secondarily - to the mitochondria. Increase 


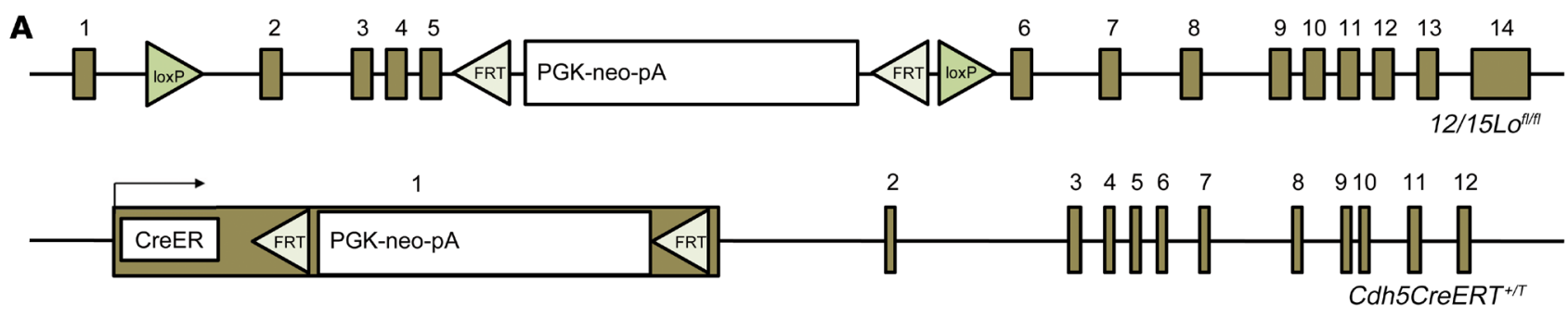

B

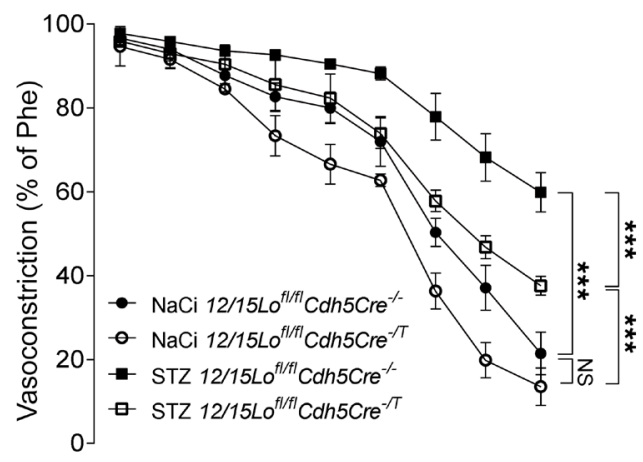

C

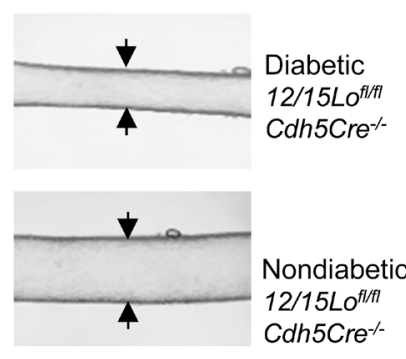

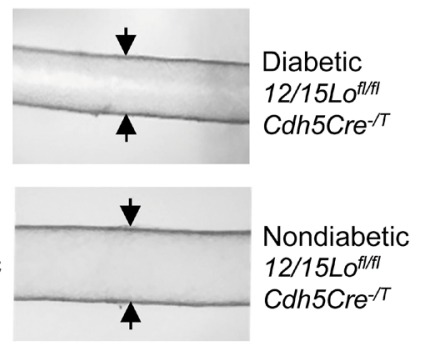

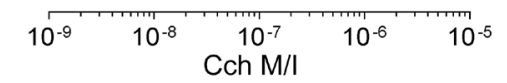

D
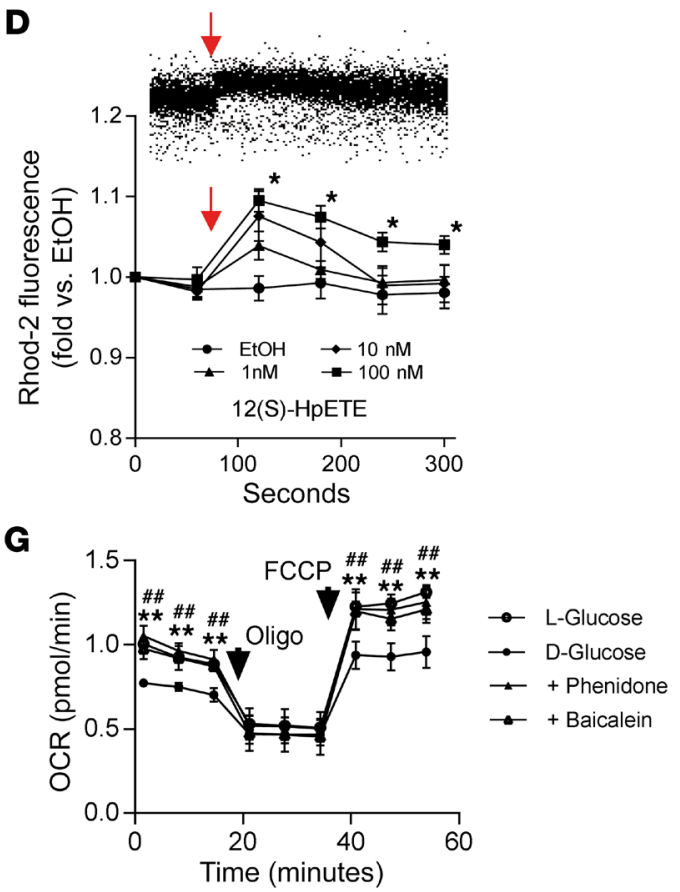

E

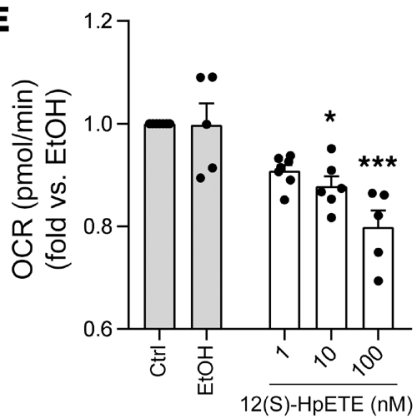

$\mathbf{F}$

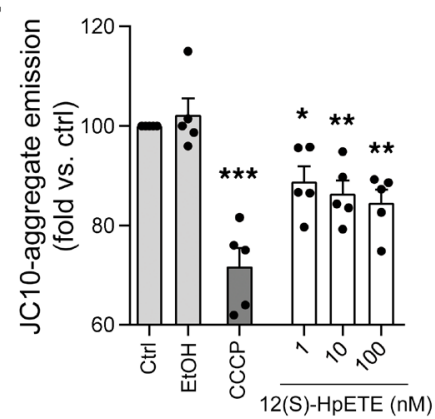

H

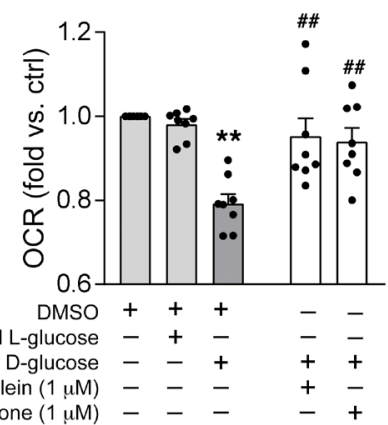

Figure 1. 12(S)-HETE mediates diabetes-induced endothelial and mitochondrial dysfunction. (A) Mice used to generate endothelium-specific 12/15Lo knockout mice. (B) Carbamoylcholine (Cch) induced vasorelaxation in murine mesenteric resistance arteries preconstricted with $10^{-5} \mathrm{M}$ phenylephrine (Phe) in $12 / 15 \mathrm{Lo} \mathrm{ofl}^{\mathrm{fl} / \mathrm{l}}$ mice positive $\left(\mathrm{Cre}^{-/ T}\right)$ or negative $\left(\mathrm{Cre}^{-/-}\right)$for the Cre transgene. Type 1 diabetes was induced by injection of streptozotocin (STZ), dissolved in sodium citrate $(\mathrm{NaCi})$ serving as vehicle control in nondiabetic mice. ${ }^{* *} P<0.001 \mathrm{vs}$. as indicated, 2 -way ANOVA/Bonferroni. $n=5$ mice per group. $\mathrm{M} / \mathrm{I}$, moles per liter. (C) Representative pictures of Cch-induced vasorelaxation at $10^{-5} \mathrm{M}$. (D) Mitochondrial calcium influx over time detected as fluorescence intensity changes by flow cytometry in rhodamine-2-loaded (Rhod-2-loaded) human endothelial cells stimulated with 12(S)-HpETE, with time of addition indicated by arrows. Upper panel shows a representative flow cytometry dot plot, lower panel the quantitative summary of $n=6$ independent experiments. Ethanol (EtOH) served as vehicle control. ${ }^{*} P<0.05$ vs. mean baseline, 2-way ANOVA/Bonferroni. (E) Mitochondrial respiration (oxygen consumption rate, OCR) measured by a Seahorse extracellular flux analyzer. ${ }^{*} P<0.05$, ${ }^{* * *} P<0.001$ vs. EtOH, 1-way ANOVA/Bonferroni, $n=5-7$ experiments per group. (F) Decline of mitochondrial membrane potential detected by flow cytometry as loss of red fluorescence. Two micromolar CCCP was used as positive control. ${ }^{*} P<0.05,{ }^{* *} P<0.01,{ }^{* *} P<0.001$ vs. EtOH, 1-way ANOVA/Bonferroni, $n=5-7$ independent experiments. (C) Extracellular flux analysis of high-glucose-exposed human endothelial cells. Inhibition of 12LOX by baicalein or phenidone improves mitochondrial OCR. Maximum mitochondrial respiration was assessed after addition of oligomycin (Oligo) and FCCP, indicated by arrows. ${ }^{*} P<0.01$ baicalein, ${ }^{* \#} P<0.01$ phenidone vs. D-glucose only, 2 -way ANOVA/Bonferroni. Pooled data from $n=5-8$ independent experiments with each variant each analyzed in triplicate. (H) Summary of basal respiration data. ${ }^{* *} P<0.01$ vs. L-glucose, ${ }^{\# \# ~} P<0.01$ vs. D-glucose, 1 -way ANOVA/Bonferroni, $n=8$ independent experiments. All graphs show mean \pm SEM. 
in mitochondrial calcium was further associated with loss of mitochondrial membrane potential and impaired mitochondrial respiration (Figure 2, F and $G$ ) that were further accompanied by impaired endothelial cell function (Supplemental Figure 5).

TRPV1 expression and function under high-glucose conditions and effects of TRPV1 deficiency in diabetic mice. To evaluate whether TRPV1 expression and function are affected by high-glucose conditions, patch clamp analysis of capsaicin-evoked responses was repeated in endothelial cells subjected to high-glucose conditions for 72 hours. In high-glucose-treated endothelial cells 10 $\mu \mathrm{M}$ capsaicin revealed induction of currents comparable to that observed previously (Figure 3A vs. Figure 2A). Western blot experiments showed that TRPV1 expression remained unchanged under high-glucose conditions in endothelial cells in vitro within 24 hours and up to 5 consecutive days (exemplarily shown in Figure 3B after 48 hours). To test whether TRPV1 deficiency is of relevance to diabetes-induced endothelial dysfunction, type 1 diabetes mellitus was induced in Trpv1-knockout and WT mice. Trpv1 knockout equally responded to streptozotocin injection with developing high glucose levels (Supplemental Figure 1), and mean 12(S)-HETE plasma levels increased more than 3-fold in diabetic compared with nondiabetic Trpv1-knockout mice $(169.6 \pm 41.6$ vs. $47.5 \pm 6.2$ $\mathrm{ng} / \mathrm{mL}, P<0.01$; Supplemental Figure 2). In contrast to diabetic WT mice, diabetic Trpv1-knockout mice exhibited preserved endothelium-dependent vasorelaxation $(P<0.001$; Figure $3 C)$ and showed no impairment of regenerative responses after arterial injury (Supplemental Figure 6). In vitro, TRPV1 activation with capsaicin induced comparable decline in mitochondrial function in human endothelial cells cultured under high-glucose conditions versus under normal-glucose conditions (Figure 3D). Antagonizing TRPV1 with the specific antagonist BCTC in endothelial cells subjected to high D-glucose augmented mitochondrial respiration, and endothelial cells isolated from Trpv1-knockout mice were protected against high-glucose-induced mitochondrial dysfunction (Figure 3, E and F), suggesting that TRPV1 inhibition or absence exerts beneficial effects for the preservation of mitochondrial and endothelial cell function under high-glucose conditions.

Importance of TRPV1 for mediating 12(S)-HETE effects. After verifying that both 12(S)-HETE and functional TRPV1 play a role in diabetes-induced endothelial dysfunction, we aimed to test the importance of TRPV1 for mediating 12(S)-HpETE effects. We first performed patch clamp experiments revealing that 12(S)-HpETE induced currents in endothelial cells comparable to those induced by the TRPV1 agonist capsaicin and that these currents could similarly be antagonized by the TRPV1 antagonist BCTC (Figure 4A). Next, we analyzed 12(S)-HpETE-evoked mitochondrial calcium influx in murine pulmonary endothelial cells isolated from WT and Trpv1-knockout mice. While 12(S)-HpETE induced mitochondrial calcium influx in endothelial cells from WT mice, these effects were absent in endothelial cells isolated from Trpv1-knockout mice (Figure 4B). Similar results were obtained regarding the effects of 12(S)-HETE on mitochondrial function, with 12(S)-HpETE effects absent in murine endothelial cells isolated from Trpv1-knockout in contrast to endothelial cells from WT mice (Figure 4C). Findings of impaired mitochondrial function further translated to endothelial function assessed in vitro as endothelial capillary-like tube formation on extracellular matrix equivalents. Here, 12(S)-HpETE impaired tube formation in endothelial cells isolated from WT but not Trpv1-knockout mice. 12(S)-HpETE effects in endothelial cells from WT mice were diminished in the presence of the TRPV1 antagonist BCTC (Figure 4, D and E), providing evidence that functional TRPV1 is required for $12(\mathrm{~S})$-HpETE to induce mitochondrial and endothelial cell dysfunction. In addition to 12(S)-HpETE, the human 12LOX and murine 12/15LO enzyme also generates 15(S)-HpETE, and 15(S)-HpETE also activates TRPV1, although with reduced potency compared with 12(S)-HpETE (13). To assess a possible role of $15(\mathrm{~S})$-HpETE in contributing to the observed effects in type 1 diabetes, 15(S)-HpETE plasma concentrations were determined in diabetic and nondiabetic mice. 15(S)-HpETE levels were 10-fold lower in nondiabetic mice compared with 12(S)-HpETE concentrations $(20.8 \pm 1.3$ vs. $239.7 \pm 60.48 \mathrm{ng} / \mathrm{mL})$ and were not elevated under diabetic conditions $(80.2 \pm 13.9 \mathrm{ng} / \mathrm{mL}$ in diabetic mice, $P=$ NS vs. nondiabetic mice; Supplemental Figure 7A). In line with the previously reported potency of 15(S)-HpETE to activate TRPV1, exogenously administered 15(S)-HpETE also triggered mitochondrial calcium influx, although less potently compared with 12(S)-HpETE in human endothelial cells. In contrast to 12(S)-HpETE, 15(S)-HpETE did not induce mitochondrial dysfunction in endothelial cells in vitro (Supplemental Figure 7, B-D).

Targeting the TRPV1 TRP box ameliorates diabetes-induced vascular pathology. Based on the findings reported above, we hypothesized that abrogation of 12(S)-HpETE-mediated TRPV1 activation can reduce high-glucose-induced mitochondrial dysfunction in endothelial cells and protect against vascular pathology in diabetes. To block 12(S)-HpETE-induced TRPV1 activation, we used a peptide constituting parts of the conserved TRP box of TRPV1 coupled to a transactivator of transcription (TAT) linker previously used to inhibit TRPV1 activation (V1-cal; Figure 5A and ref. 23). Patch clamp analysis confirmed that 12 (S)-HpETE was unable to evoke currents in V1-cal-loaded human endothelial cells in contrast to the 12(S)-HpETE response detected in cells loaded with TAT linker protein (Figure 5B). Preincubation with V1-cal abolished 12(S)-HpETE-induced mitochondrial calcium influx in contrast to 12(S)-HpETE effects in endothelial cells preincubated with the TAT linker protein or a scrambled version of V1-cal (V1-scr) coupled to TAT used as controls (Figure 5C). V1-cal but not TAT or V1-scr reduced 12(S)-HpETE-induced decrease of mitochondrial respiration (Figure 5D) and abolished the effects of 12(S)-HpETE that impaired endothelial cell capillary-like tube formation (Supplemental Figure 8). Notably, neither V1-cal, the TAT linker protein, nor V1-scr altered 12LOX expression in human endothelial cells in vitro (Supplemental Figure 9). In vivo, injection of V1-cal, TAT, or V1-scr for 4 consecutive days 4 weeks after streptozotocin injection revealed that V1-cal but not TAT or V1-scr was able to improve endothelial dysfunction in diabetic mice (Figure 5E). Continuous delivery of V1-cal via subcutaneously implanted osmotic minipumps for 3 weeks after arterial injury in diabetic mice improved vascular regeneration compared with histomorphometrically assessed quantitative measures of nondiabetic control mice (Supplemental Figure 10). In V1-caltreated diabetic mice, 12(S)-HETE concentrations were undetectable in murine plasma, possibly owing to formation of complexes of 12(S)-HpETE with V1-cal. 
A
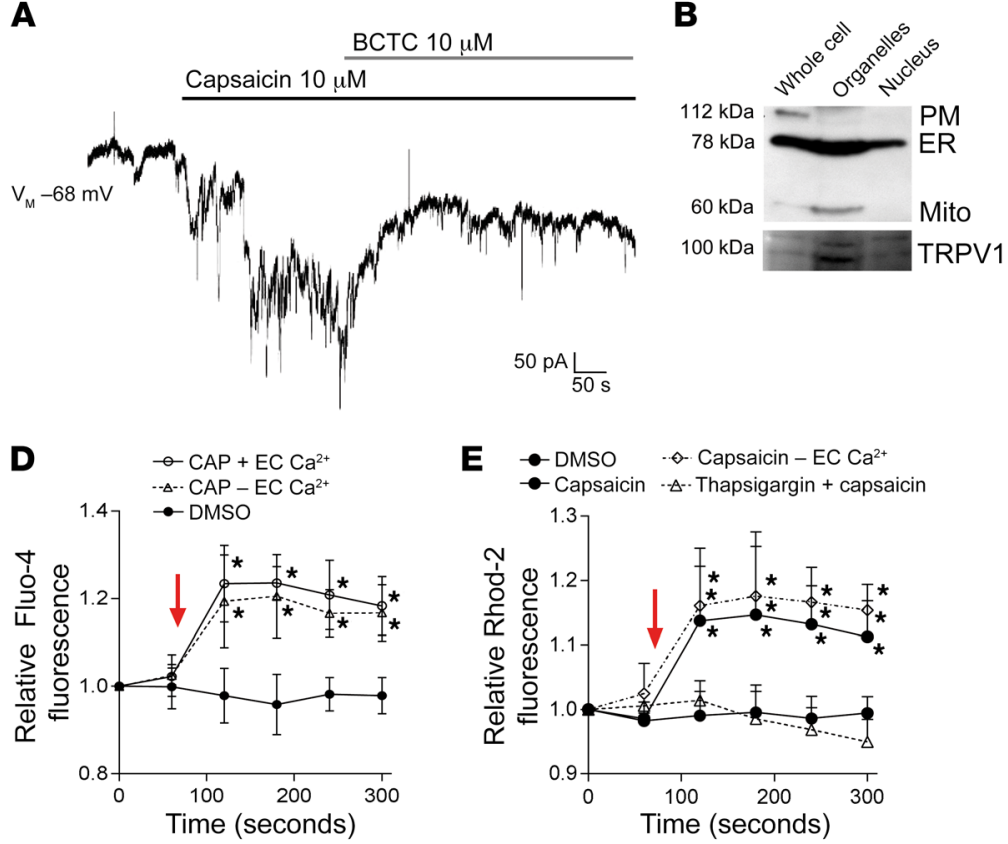

E - DMSO $\diamond$ Capsaicin- $\mathrm{EC} \mathrm{Ca}^{2+}$

- Capsaicin - - - Thapsigargin + capsaicin

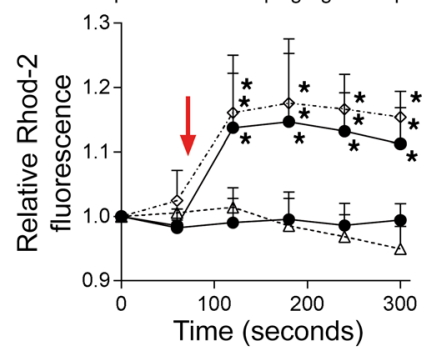

C

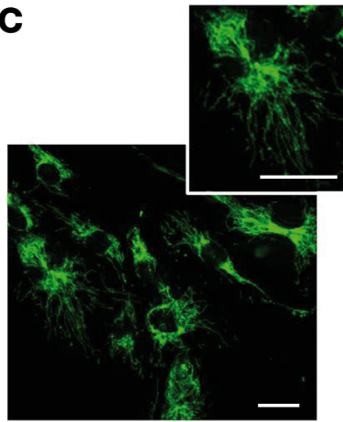

Tom20

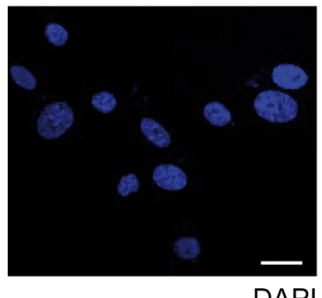

DAPI

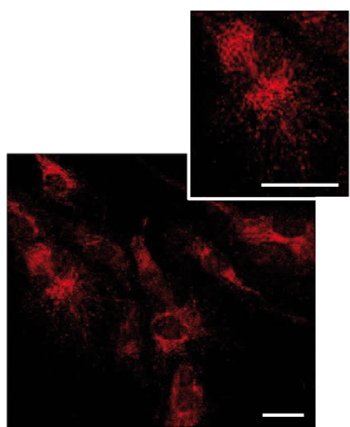

TRPV1

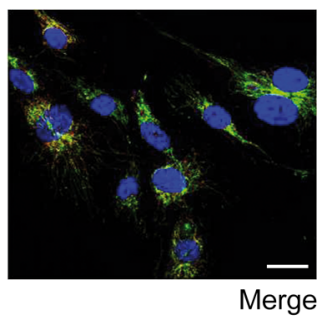

$\mathbf{F}$

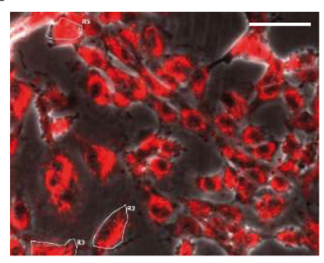

$0 \mathrm{~min}$

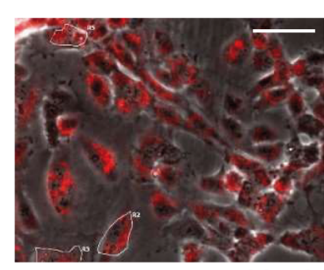

$30 \mathrm{~min}$
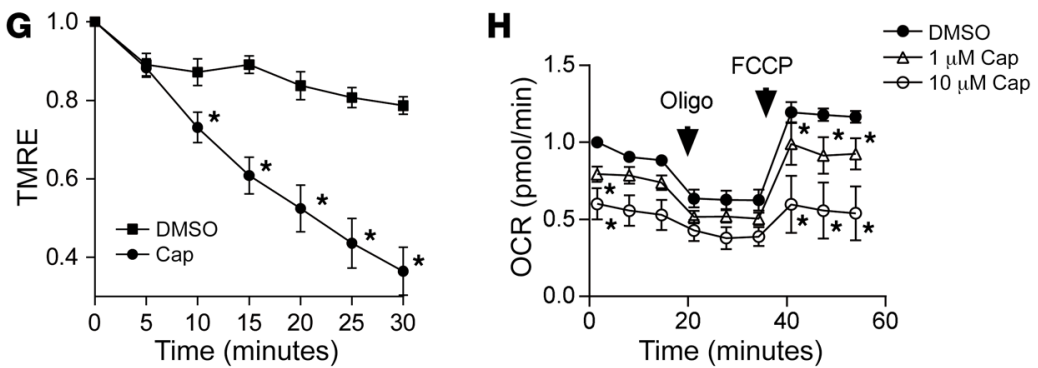

Figure 2. TRPV1, a receptor activated by 12(S)-HpETE, is present and functional in human endothelial cells, located intracellularly, and a mediator of mitochondrial dysfunction. (A) Representative patch clamp experiment of human endothelial cell exposure to $10 \mu \mathrm{M}$ capsaicin shows currents induced by TRPV1 activation that are antagonized by $10 \mu \mathrm{M}$ of the TRPV1 antagonist BCTC. Representative patch of $n=15$ independent experiments. (B) Cell fractionation reveals that TRPV1 is enriched in fractions containing ER (here identified by GRP78 expression) and mitochondria (Mito, ATP5a). PM, plasma membrane, $\mathrm{Na}^{+} / \mathrm{K}^{+}$-ATPase. (C) Immunohistochemistry showing that TRPV1 (red) colocalizes with Tom20 (green) in human endothelial cells. Cell nuclei are stained with DAPI. Scale bars: $20 \mu \mathrm{m}$. (D and E) Ten micromolar capsaicin (time point of addition indicated by arrow) induces increase in cytosolic calcium in Fluo-4-loaded HUVECs and increase in mitochondrial calcium in Rhod-2-loaded HUVECs independent of the presence (+ EC Ca $\left.{ }^{2+}\right)$ or absence $\left(-\mathrm{EC} C \mathrm{a}^{2+}\right)$ of extracellular calcium but dependent on calcium in the ER since effects were absent after thapsigargin-induced ER depletion. ${ }^{*} P<0.01$ vs. baseline fluorescence, 2-way ANOVA/Bonferroni, $n=6$ independent experiments. (F) One micromolar capsaicin induces decline in mitochondrial membrane potential indicated as decline in fluorescence intensity of TMRE-loaded human endothelial cells during live-cell imaging. ${ }^{*} P<0.05$ vs. baseline fluorescence intensity, 2-way ANOVA/Bonferroni, mean of at least 5 cells per high-power field in $n=5$ independent experiments. Scale bars: $50 \mu$ m. (G) Prestimulation of human endothelial cells with $1 \mu \mathrm{M}$ or $10 \mu \mathrm{M}$ capsaicin reduces mitochondrial OCR. Maximum mitochondrial respiration was assessed after addition of oligomycin and FCCP, indicated by arrows. ${ }^{*} P<0.05$ vs. baseline, 2-way ANOVA/Bonferroni. Pooled data from $n=5$ independent experiments with each variant analyzed in triplicate. All graphs show mean \pm SEM.

\section{Discussion}

Type 1 and type 2 diabetes mellitus inevitably lead to vascular disease that almost exclusively determines the multiple comorbidities related to diabetes. Despite the implementation of traditional strategies to lower cardiovascular risk, diabetic patients have a 7 to 10 times higher risk of suffering from adverse cardiovascular events (24), demanding a more specific understanding of vascular disease pathology in diabetes. We here identify that elevated plasma concentrations of 12(S)-HETE directly link the hyperglycemic condition to vascular disease initiation in diabetes. We show that 12(S)-HpETE induces mitochondrial calcium increase and mitochondrial dysfunction characteristic of endothelial cells from diabetic patients. We identify the cation channel TRPV1 at a previously unrecognized intracellular localization in endothelial cells where it transmits 12(S)-HpETE effects at the mitochondria. By targeting 12(S)-HpETE interaction with TRPV1 at the TRPV1 C-terminal TRP box, we uncovered a potential pharmacological strategy that could allow causal control of diabetes-induced vascular disease initiation and progression.

One of the central problems in the clinical management of diabetic patients is poor glycemic control. Both chronic hyperglycemia and repeated hyperglycemic episodes increase endothelial oxidative metabolism and result in reduced bioavailability of the endothelium-derived vasodilator nitric oxide. Impaired endothelium- 
A

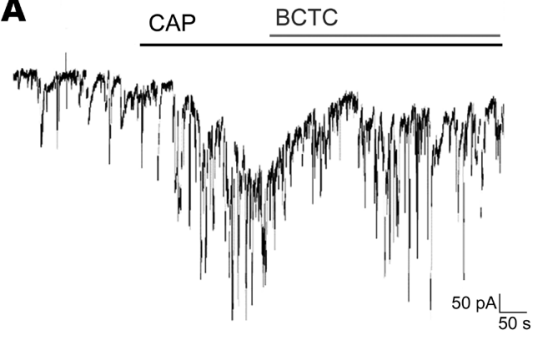

B
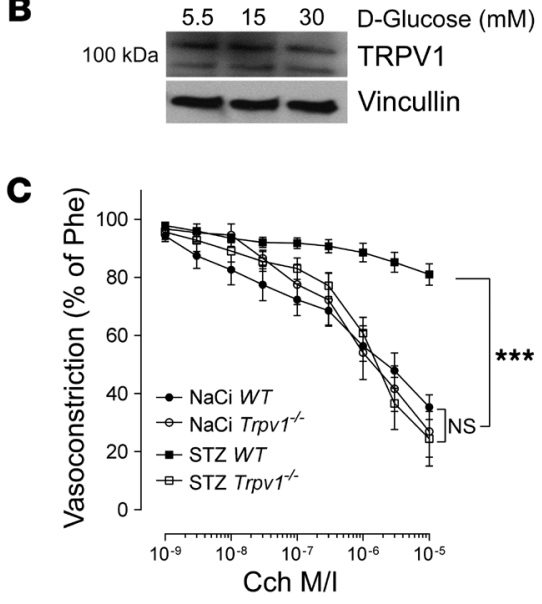
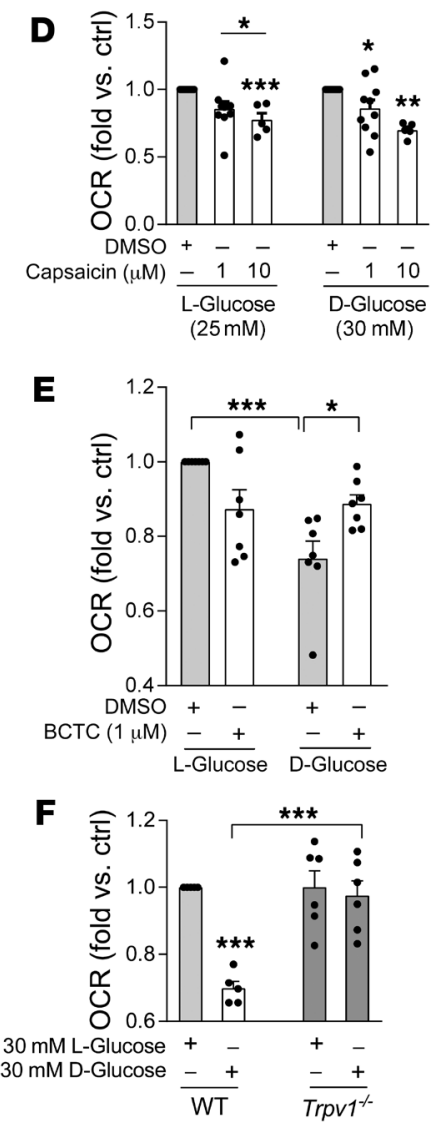

Figure 3. TRPV1 presence and function are unaltered under high-glucose conditions and TRPV1 deficiency protects against diabetes-induced endothelial dysfunction. (A) Patch clamp experiments of human endothelial cells cultured under high levels of D-glucose (30 mM) show robust currents induced by $10 \mu \mathrm{M}$ of capsaicin (CAP) that are antagonized by $10 \mu \mathrm{M}$ of the TRPV1 antagonist BCTC. Representative result out of $n=$ 10 independent experiments. (B) Representative Western blot of human endothelial cells cultured under high-glucose conditions for $24-96$ hours (here 48 hours) shows that TRPV1 expression is not altered by high glucose. Twenty-five or $10 \mathrm{mM} \mathrm{D}$-glucose was added to $5.5 \mathrm{mM} \mathrm{o}$-glucose in media. Twenty-five or $15 \mathrm{mM}$ L-glucose was added for osmotic control. (C) Cch-induced vasorelaxation in murine mesenteric resistance arteries preconstricted with $10^{-5} \mathrm{M}$ phenylephrine shows that TRPV1-knockout ( $\left.\operatorname{Trpv1}^{-/-}\right)$mice are protected from diabetes-induced endothelial dysfunction. STZ, streptozotocin; $\mathrm{NaCi}$, sodium citrate serving as vehicle control. ${ }^{* *} P<0.001$ vs. as indicated, 2 -way ANOVA/ Bonferroni. Means were generated for each individual animal by analysis of 2 arteries, $n=10$ animals per group. (D) Capsaicin reduces mitochondrial OCR in a comparable fashion in endothelial cells exposed to high levels of L- or D-glucose. ${ }^{*} P<0.05$, ${ }^{* *} P<$ $0.01,{ }^{* *} P<0.001$ vs. DMSO or as indicated, 1-way ANOVA/Bonferroni, summary of $n=$ 5-10 independent experiments. (E) Impaired mitochondrial respiration under high-Dglucose conditions ( $30 \mathrm{mM}$ ) in human endothelial cells is improved by coincubation with the TRPV1 antagonist BCTC. ${ }^{*} P<0.05,{ }^{* * *} P<0.001$ vs. as indicated, 1-way ANOVA/ Bonferroni, $n=7$ independent experiments. (F) High levels of D-glucose impair mitochondrial respiration in endothelial cells isolated from WT but not Trpv1 $7^{-1-}$ mice. Endothelial cells were analyzed from $n=5-6$ independent isolations. ${ }^{* *} P<0.001 \mathrm{vs}$. L-glucose or as indicated. All graphs show mean \pm SEM.

dependent vasodilation can be assessed and quantified in diabetic patients and is an independent marker of cardiovascular risk and predictor of future adverse cardiovascular events (25). Endothelial dysfunction in diabetes is crucially connected to disturbance in intracellular calcium homeostasis and loss of mitochondrial integrity, but the mechanisms are incompletely understood $(8,26)$. In response to high glucose, endothelial cells upregulate 12LOX that

metabolizes arachidonic acid to bioactive lipid mediators such as 12(S)-HpETE. Based on the observation that mice deficient in $12 / 15 \mathrm{Lo}$, the mouse equivalent of human 12LOX, are resistant to chemical and high-fat diet-induced diabetes, 12LOX metabolites have been identified as mediators of oxidative stress in pancreatic $\beta$ cell dysfunction during diabetes development. 12LOX inhibitors are currently discussed as pharmacological tools to limit diabetes initiation and possibly the complications related to disease progression (27). In the cardiovascular system, a correlation of 12LOX activity with reduced nitric oxide bioavailability and a proatherogenic phenotype has been well characterized in mouse models of 12/15Lo knockout and overexpression (reviewed in Gleissner et al., ref. 28).

To specify the role of 12LOX and its metabolites in the endothelium, we here generated mice with an inducible and endothelium-specific deletion of 12/15Lo. When diabetic, these mice showed no increase in 12(S)-HETE plasma concentration compared with their WT diabetic littermates. Since 12(S)-HpETE and its stable metabolite 12(S)-HETE can freely diffuse across cell membranes (29), our results suggest that endothelial cells are a major contributor to the increased 12(S)-HETE plasma concentrations in diabetic patients.

Although our findings thus mechanistically support the role for increased 12LOX metabolites in diabetic patients with renal and coronary vascular disease (11, 12), further studies are required to determine the exact source of increased 12(S)-HETE levels in diabetic mice in comparison with diabetic humans. Despite the presence of high glucose levels, diabetic mice with endothelial 12/15Lo knockout exhibited preserved endothelium-dependent vasodilation. In addition, they showed uncompromised vascular regenerative responses to arterial injury in contrast to their diabetic WT littermates, in which increased neointima formation was present 3 weeks after arterial injury. For arterial injury induction, we topically applied ferric chloride to the carotid artery that penetrates the vessel wall and causes endothelial denudation (30). After the initial thrombus formation and resolution, vascular repair is critically determined by re-endothelialization (31). Although we cannot extract specific cellular or molecular mechanistic insights into the underlying pathophysiology, these results can further support the notion that endothelial cell function in diabetic mice is sustained by 12/15Lo deletion. Already 30 years ago, hydroxyeicosatetraenoic acids were implicated in the mobilization of mitochondrial calcium, but a clinical implication of these findings remained unidentified (32). Here, we observed that 12(S)-HETE concentrations corresponding to those found in patients with diabetes (ranging from $\sim 30 \mathrm{nM}$ in ref. 11 to $\sim 300$ $\mathrm{nM}$ in ref. 10) increased mitochondrial calcium levels in human and murine endothelial cells, and these effects were associated with loss of mitochondrial membrane potential and mitochondrial dysfunction, the hallmarks of diabetes-induced endothelial 
A

12(S)-HpETE BCTC

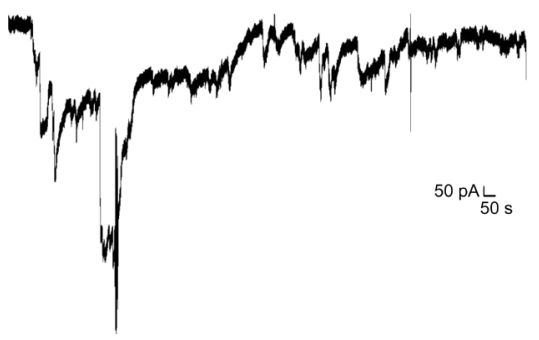

B $\quad-\bullet W T \quad-\because \cdot 1 \mu \mathrm{M} 12(\mathrm{~S})-\mathrm{HpETE}$
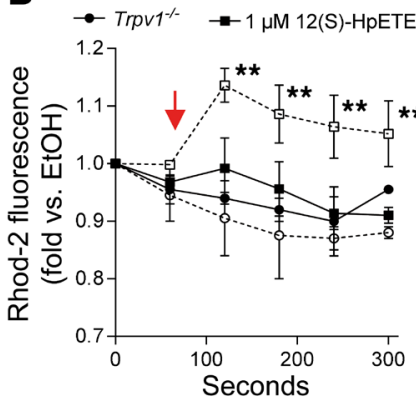

C

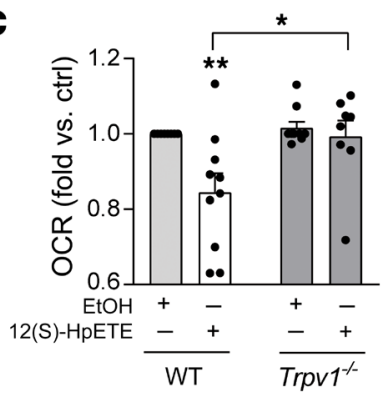

D

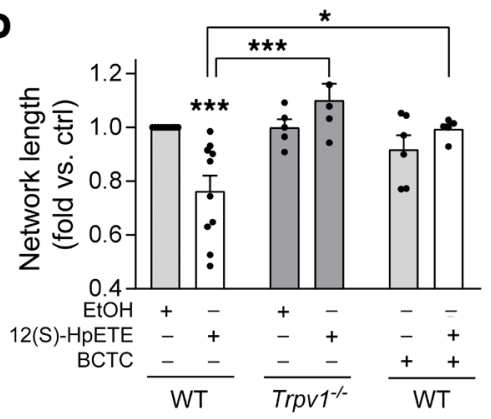

Figure 4. 12(S)-HpETE effects on mitochondrial and endothelial function are mediated by TRPV1. (A) Patch clamp experiments with human endothelial cells show that $700 \mathrm{nM}$ 12(S)-HpETE induces currents comparable to those induced by $10 \mu \mathrm{M}$ capsaicin and that these currents are antagonized by $10 \mu \mathrm{M}$ of the TRPV1 antagonist BCTC. (B) Mitochondrial calcium increase induced by $1 \mu \mathrm{M} 12(\mathrm{~S})$-HpETE added as indicated by the arrow and assessed as increase in fluorescence intensity of Rhod-2-loaded murine endothelial cells isolated from WT (dashed lines) versus Trpv1 $1^{-/-}$mice. ${ }^{* *} P<$ 0.01 vs. baseline, 2-way ANOVA/Bonferroni, summary of $n=$ 5 independent experiments. (C) Mitochondrial OCR is reduced by $100 \mathrm{nM} 12(\mathrm{~S})$-HpETE in endothelial cells from WT but not Trpv1 ${ }^{-1-}$ mice. ${ }^{*} P<0.05,{ }^{* *} P<0.01 \mathrm{vs}$. EtOH or as indicated, 1-way ANOVA/Bonferroni, $n=5-10$ independent experiments (D and $\mathbf{E}$ ) Effects of 12(S)-HpETE on endothelial capillary-like tube formation on Matrigel. Effects of $100 \mathrm{nM} 12$ (S)-HpETE are absent in murine endothelial cells isolated from Trpv1 ${ }^{-1-}$ mice and endothelial cells isolated from WT mice in the presence of $10 \mu \mathrm{M}$ of the TRPV1 antagonist BCTC. ${ }^{*} P<0.05$, ${ }^{* *} P$ $<0.001$ vs. EtOH or as indicated, 1-way ANOVA/Bonferroni, $n=5-10$ independent experiments. Scale bar: $500 \mu \mathrm{m}$. All data are presented as mean \pm SEM.
E

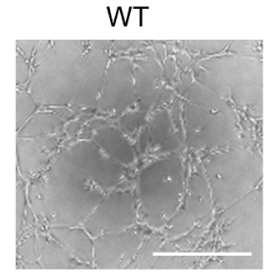

EtOH

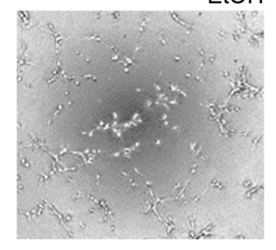

12(S)-HpETE

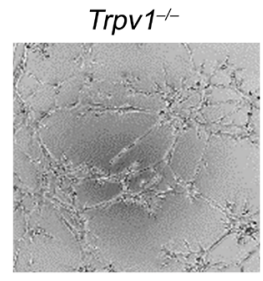

$\mathrm{EtOH}$

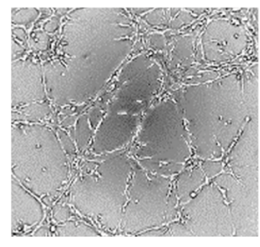

12(S)-HpETE

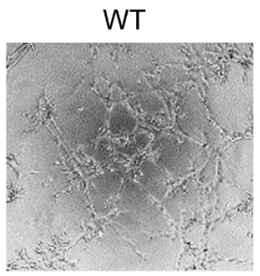

$\mathrm{BCTC}$

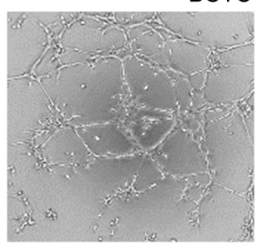

12(S)-HpETE + BCTC dysfunction. Our results thus suggest that 12(S)-HETE is a direct mediator of endothelial dysfunction in diabetes and that increased 12(S)-HETE plasma levels could serve as a biomarker reflecting the degree of mitochondrial impairment, endothelial dysfunction, and thus vascular disease severity in patients with diabetes.

A known receptor of 12(S)-HETE is $G$ protein-coupled receptor 31 (GPR31), and the 12(S)-HETE/GPR31 signaling axis is implicated in cancer metastasis and ischemia/reperfusion injury (33). While one study reported the presence of GPR31 in human endothelial cells at the mRNA level (34), we and others were unable to detect GPR31 in endothelial cells or the vasculature (35). In addition, 12(S)-HpETE effects on mitochondrial calcium influx and mitochondrial function were unaffected by the presence of pertussis toxin, suggesting that a $G$ protein-coupled receptor is not involved in the effects of 12(S)-HpETE reported here (Supplemental Figure 11). In line with these results, the role of 12(S)-HETE in diabetes has been suggested to be mediated by other receptor molecules (33), which is further supported by reports that binding of 12(S)-HpETE to GPR31 is unable to evoke changes in intracellular calcium (36).
In addition to GPR31, 12(S)-HpETE is a known agonist of TRPV1 and activates TRPV1 with a $K_{I}$ of $350 \mathrm{nM}(\sim 117 \mathrm{ng} / \mathrm{mL})$, a concentration found mostly in diabetic, not nondiabetic, rodents and humans. TRPV1 mediates increase in intracellular calcium levels in response to activation by capsaicin binding to intracellular localizations $(14,37)$. We here verified TRPV1 expression and function in endothelial cells by patch clamp analysis, Western blot, immunohistochemistry, and calcium flux analysis. Although TRPV1 is considered to primarily localize at the plasma membrane, immunohistochemistry of endothelial cells unraveled an intracellular localization of TRPV1 that was identical to the staining pattern of endothelial mitochondria. TRPV1 activation using capsaicin as agonist revealed that TRPV1 mobilizes calcium from intracellular calcium stores such as the endoplasmic reticulum (ER) that results in cytosolic calcium increase and - primarily or secondarily - in mitochondrial calcium uptake and increased mitochondrial calcium content. Intracellular localizations of TRPV1 have been identified previously, and a fundamental role of TRPV1 in regulating intracellular calcium has been proposed (16). In the regulation of 
A

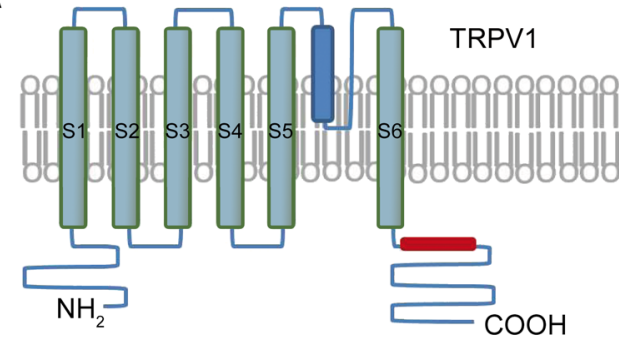

V1-cal: TAT + RAITILDTEKS

C

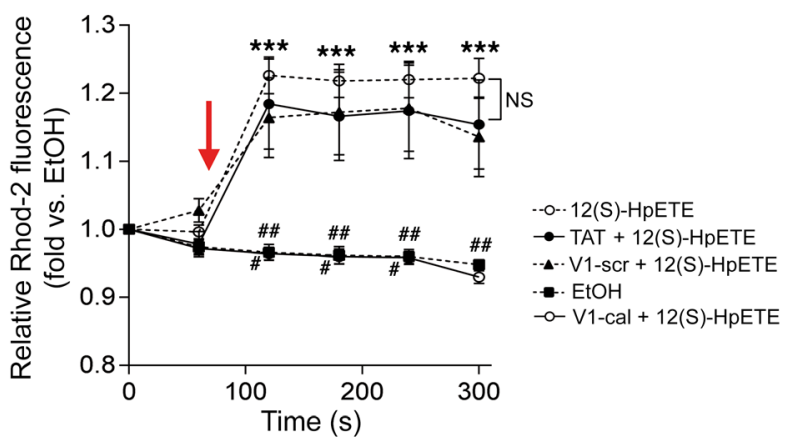

E

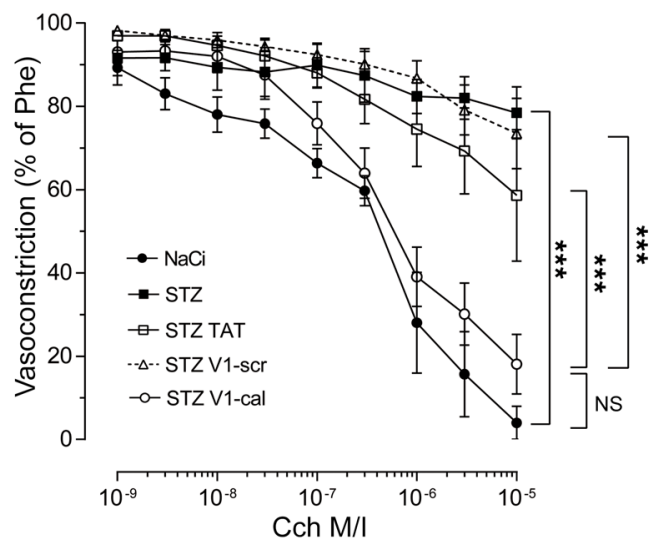

B

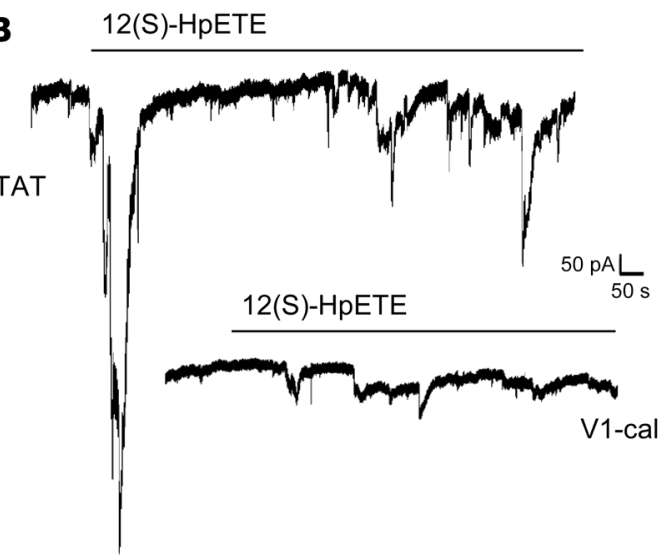

D
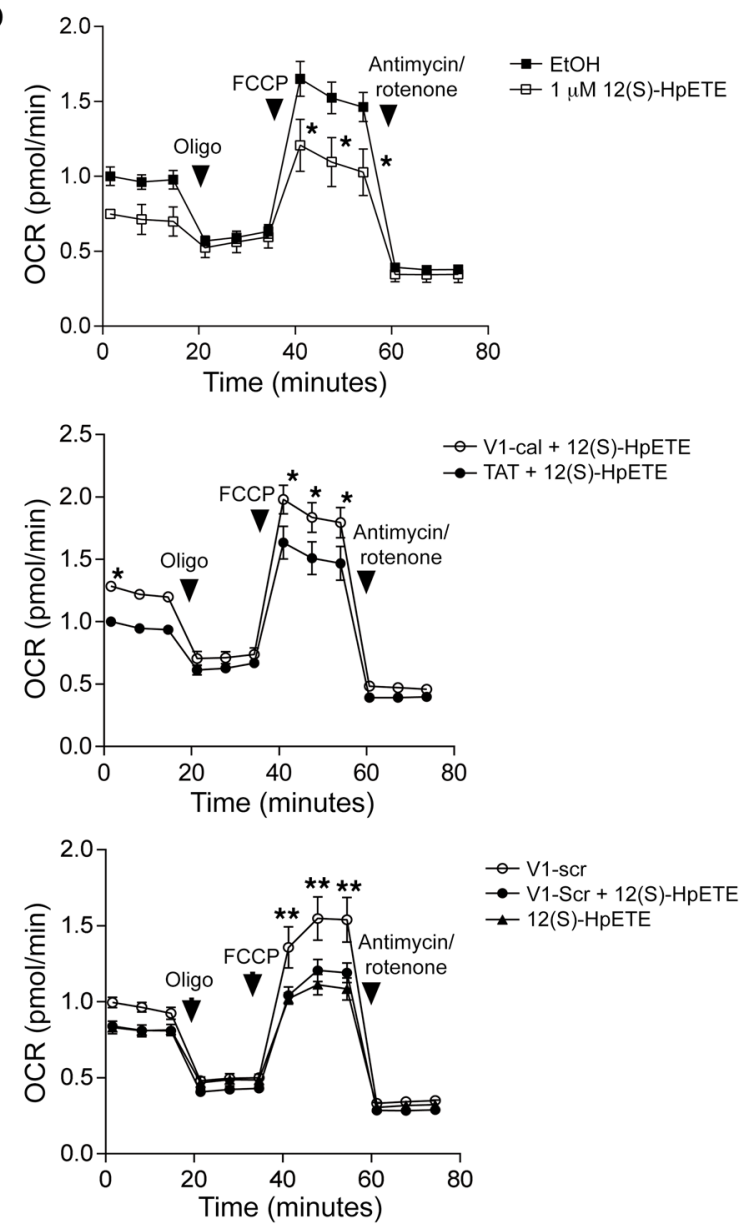

Figure 5. Abrogation of the 12(S)-HpETE/TRPV1 interaction protects against diabetes-induced endothelial dysfunction. (A) Schematic of TRPV1 corresponding to amino acids 701-711 within the TRP box at the C-terminus of human TRPV1 linked to a TAT linker protein for intracellular entry. (B) Patch clamp experiments show that $700 \mathrm{nM} 12(\mathrm{~S})$-HpETE evokes currents in endothelial cells loaded with $1 \mu \mathrm{M}$ TAT linker, but not $1 \mu \mathrm{M}$ V1-cal. Representative patch results from $n=10$ independent experiments. (C) 12(S)-HpETE-induced mitochondrial calcium changes detected by flow cytometry [time point of addition of 12(S)-HpETE is indicated by the arrow] in human endothelial cells preincubated with either $1 \mu \mathrm{M}$ V1-cal or $1 \mu \mathrm{M}$ of a scrambled version of V1-cal (V1-scr) or TAT linker protein

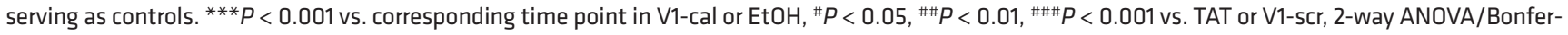
roni, $n=5$ independent experiments. (D) Assessment of mitochondrial function to evaluate effects of $1 \mu \mathrm{M}$ V1-cal versus $1 \mu \mathrm{M}$ TAT linker only (top 2 panels) or $1 \mu \mathrm{M}$ V1-scr (bottom panel) on mitochondrial OCR induced by $1 \mu \mathrm{M} 12$ (S)-HpETE. After 3 measurements, oligomycin was added before FCCP used to induce maximum respiration. ${ }^{*} P<0.05,{ }^{*} P<0.01,2$-way ANOVA/Bonferroni. Variants were analyzed in triplicate on Seahorse miniplates, and $n=5$ independent experiments were performed. All graphs show mean \pm SEM. (E) Cch-induced vasorelaxation shows that repetitive intravenous application on 4 consecutive days of the V1-cal peptide $(1 \mathrm{mg} / \mathrm{kg} / \mathrm{d})$, but not of the V1-scr peptide or the TAT linker protein only, to diabetic WT mice reduced endothelial dysfunction induced by diabetes (STZ). Sodium citrate (NaCi) was used as vehicle control. ${ }^{* *} P<0.001$ vs. as indicated, 2-way ANOVA/Bonferroni. Means were generated for each individual animal by analysis of 2 arteries; $n=5$ animals per group. Graph shows mean \pm SEM. 
nociception, crosstalk specifically between TRPV1 and the mitochondrial calcium shuttling machinery was previously identified (38). In obesity-induced mitochondrial dysfunction, mitochondrial calcium increase is mediated by an increased formation of mitochondria-associated membranes (MAMs) and thus mitochondria/ ER interactions that facilitate mitochondrial calcium overload and result in metabolic dysfunction (39).

Although future investigations will have to clarify the exact localization of TRPV1 in relation to the mitochondria, our analysis of the functional consequences of TRPV1 activation revealed that TRPV1 stimulation by capsaicin induces changes to mitochondrial function identical to those induced by 12(S)-HpETE and high levels of glucose. Our results further show that Trpv1-knockout mice are equally as protected from diabetes-induced endothelial dysfunction and impaired responses to arterial lesions in diabetes as are mice with endothelial $12 / 15 \mathrm{Lo}$ knockout. Further analysis showed that 12(S)-HpETE effects were abolished in the presence of BCTC, a well-established TRPV1 antagonist (40), or in endothelial cells isolated from Trpv1-deficient mice, and both BCTC and Trpv1 deletion can preserve mitochondrial function under high-glucose conditions. Under high-glucose conditions, TRPV1 expression in endothelial cells was not affected. Contrary to possible desensitization, high-glucose conditions are reported to rather sensitize TRPV1, resulting in increased agonist-evoked calcium responses in diabetes $(41,42)$. In dorsal root ganglia, increased TRPV1 sensitivity is suggested to link to early painful diabetic neuropathy (43), further supporting the assumption that TRPV1 signaling is not compromised under diabetic conditions. We thus propose TRPV1 as a central mediator of increased 12(S)-HETE concentrations mediating mitochondrial dysfunction and vascular pathology in diabetes.

In acute scenarios such as ischemia/reperfusion injury, TRPV1 may also confer protection from intracellular localizations and involvement in cellular calcium homeostasis (44). Thus, antagonizing TRPV1 may not be a suitable approach for limiting TRPV1-mediated vascular pathology in diabetes because of possible side effects (45). The interaction site of 12(S)-HpETE with TRPV1 has not been identified so far. Other endogenous agonists sharing lipid structure activate TRPV1 by interacting with a positively charged lysine (K) located at site 710 of the highly conserved C-terminal TRP box of TRPV1 (46). Here, we used a peptide (V1-cal) previously characterized as a potent TRPV1 inhibitor (23) to serve as a peptide decoy for 12(S)-HpETE by mimicking the putative 12(S)-HpETE interaction site-spanning region at the C-terminal end of TRPV1. Notably, in diabetic mice subjected to application of this peptide but not the TAT linker protein, we were unable to detect 12 (S)-HETE by immunoassay, suggesting a direct interaction of V1-cal with 12(S)-HETE. Application of V1-cal-induced protection from diabetes induced endothelial dysfunction and augmented vascular regeneration after arterial injury. TRPV1 is highly conserved among species; however, a natural variant of site 710 exists in avian TRPV1 (exhibiting an uncharged asparagine, $\mathrm{N}$, instead of a positively charged $\mathrm{K}$, ref. 47). This is of particular interest since birds naturally exhibit hyperglycemia (up to $500 \mathrm{mg} / \mathrm{dL}$ ) (48) to meet the energy demands of flying long distances. We thus speculate that the 12(S)-HETE/TRPV1 interaction site is part of a protective mechanism in birds against the deleterious effects of chronically high levels of plasma glucose. Our data suggest that the interruption of 12(S)-HETE/TRPV1 interaction can represent a pharmacological strategy to preserve vascular integrity under high-glucose conditions.

Taken together, our results characterize increased plasma concentrations of 12(S)-HETE in diabetic patients as a direct mechanistic link to disturbed endothelial calcium homeostasis, mitochondrial pathology, and impaired endothelium-dependent vasodilation. We identify intracellular TRPV1 as the primary mediator of 12(S)-HETE-induced calcium changes and mitochondrial pathology in endothelial cells and suggest that abrogation of 12(S)-HETE/TRPV1 interaction at the TRPV1 TRP box can preserve endothelial cell function in diabetes. This study may support the use of 12(S)-HETE as a biomarker indicating vascular disease initiation, progression, and severity in diabetic patients and identifies a pharmacological strategy to causally control vascular disease and lower the incidence of comorbidities in patients with diabetes.

\section{Methods}

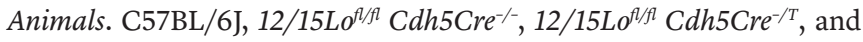
$\mathrm{Trpv1}^{-/-}$(provided by Jan Siemens, Institute of Pharmacology, Heidelberg, Germany) mice were housed on a 12-hour light/12-hour dark cycle with ad libitum access to food and water in a specified pathogenfree facility. Endothelium-specific knockout was induced in $12 / 15 \mathrm{Lo} / \mathrm{flfl}$ $\mathrm{Cdh} 5 \mathrm{Cre}^{-/ T}$ by injection of 3- to 4 -week-old mice with $75 \mathrm{mg} / \mathrm{kg}$ tamoxifen daily over 3 consecutive days. Genotypes of $12 / 15 \mathrm{Lo}^{f / / f l} \mathrm{Cdh} 5 \mathrm{Cre}^{-/-}$, 12/15Lo $0^{f / f l} \mathrm{Cdh} 5 \mathrm{Cre}^{-/ T}$, and $\operatorname{Trpv1} 1^{-/-}$were confirmed via PCR and gel electrophoresis using the following primers: $12 / 15$ Lo loxP: up 5 '-CATGGGTTCTCTGCCTTAGTGG-3', down 5'-TCACCTCAACCAAGAGTGAGAGGG-3'; Cre: up 5'-GCCTGCATTACCGGTCGATGCAACGA-3', down 5'-GTGGCAGATGGCGCGGCAACACCATT-3'; Trpv1: up 5'-CCTGCTCAACATGCTCATTG-3', up 5'-TCCTCATGCACTTCAGGAAA-3', down 5'-CACGAGACTAGTGAGACGTG-3'.

Diabetes model. For the induction of type 1 diabetes mellitus, male and female 6- to 8-week-old C57BL/6J, 12/15Lo $o^{f / f l} \mathrm{Cdh} 5 \mathrm{Cre}^{+/+}, 12 / 15 \mathrm{Lo} o^{f / f l}$ $\mathrm{Cdh} 5 \mathrm{Cre}^{+/ T}$, and $\mathrm{Trpv1}^{-/-}$mice were injected i.p. with a single dose of streptozotocin $(180 \mathrm{mg} / \mathrm{kg}$ in $0.1 \mathrm{M}$ sodium citrate, $\mathrm{pH} 4.5)$. Injection of sodium citrate only served as vehicle control. Induction of diabetes was confirmed by measurement of nonfasted blood glucose levels of at least $200 \mathrm{mg} / \mathrm{dL} 72$ hours after injection using an Accu-Check Performa glucometer (Roche). Blood plasma samples were obtained from the facial vein on the day of sacrifice and stored at $-80^{\circ} \mathrm{C}$ pending analysis. 12(S)-HETE and 15(S)-HETE levels were assessed by ELISA (ADI-900-050 and ADI-900-051, Enzo Life Sciences).

Pressure myography. For the assessment of endothelial function, third-order mesenteric resistance arteries were dissected 4 weeks after diabetes induction and mounted on glass cannulas in a vessel chamber (Living Systems Instrumentation) in calcium-free buffer (10 mM HEPES, $140 \mathrm{mM} \mathrm{NaCl}, 5 \mathrm{mM} \mathrm{KCl}, 1.2 \mathrm{mM} \mathrm{MgCl}, 10 \mathrm{mM}$ glucose, $1 \mathrm{mM}$ EGTA). Experiments were performed in calcium-containing buffer (Ca-free buffer without EGTA including $2 \mathrm{mM} \mathrm{CaCl}_{2}$ ). After the development of stable myogenic tone at $80 \mathrm{mmHg}$, vessels were exposed to $10 \mathrm{nM}(\mathrm{R})-(-)$-phenylephrine hydrochloride (Tocris) for maximum constriction. Relaxation response to increasing concentrations of Cch $\left(10^{-9}\right.$ to $10^{-5} \mathrm{M}$; Tocris) was assessed using digital video edge detection. Dependence of carbamoylcholine effects on intact endothelium and endothelium-dependent nitric oxide release were verified in each vessel by application of $100 \mu \mathrm{M} N^{\omega}$-nitro-L-arginine 
methyl ester (L-NAME; Tocris) and by analysis of a subset of vessels after endothelial denudation using an air bubble.

Carotid artery injury. Two weeks after diabetes induction or vehicle control treatment, mice were anesthetized i.p., and unilateral chemical injury of the common carotid artery was induced by exposure of arteries to $3 \%$ ferric chloride for 3 minutes as previously described (49). Mice were sacrificed 3 weeks after surgery, and the left carotid artery was removed and fixed in $4 \%$ formaldehyde. Vessels were paraffin-embedded, and cross sections performed every $5 \mu \mathrm{m}$ were stained with H\&E for morphological assessment by a blinded observer using $\times 20$ magnification on a Nikon Eclipse Ts 2 microscope. The section exhibiting the largest neointimal lesion was selected for quantitative analysis assessing neointimal area and luminal stenosis using Image J software (version 1.52a, NIH).

Peptide application. The V1-cal peptide and V1-scr, a scrambled version of V1-cal, were synthesized as 1 polypeptide with transactivator of transcription (TAT) ${ }_{47-57}$ carrier in the following order: $\mathrm{N}$-terminus- $\mathrm{TAT}_{47-57}$-spacer (Gly-Gly)-cargo-C-terminus, as described previously (23) (RAITILDTEKS, V1-cal; and IDKLRTAEIST, V1-scr). For assessment of endothelial function, mice were injected i.v. with $1 \mathrm{mg} / \mathrm{kg} / \mathrm{d}$ peptide or equimolar concentrations of TAT 4 weeks after diabetes induction for 4 consecutive days before sacrifice. For neointima assessment, $100 \mu \mathrm{L}$ of $7.5 \mathrm{mg} / \mathrm{mL}$ peptide or equimolar concentrations of TAT protein were loaded into Alzet osmotic minipumps (model 1004) for delivery of $1 \mathrm{mg} / \mathrm{kg} / \mathrm{d}$ and were subcutaneously implanted following arterial injury.

Endothelial cells, in vitro high-glucose protocol, tube formation assay, and immunohistochemistry. HUVECs (PromoCell) were cultured in attachment factor-coated dishes (Gibco, Thermo Fisher Scientific) in Endothelial Cell Growth Medium 2 kit (C-22111, PromoCell) supplemented with Endothelial Cell Growth SupplementPack (C-39211, PromoCell) and $1 \%$ penicillin-streptomycin (MilliporeSigma). Cells were passaged using Accutase (MilliporeSigma) and used from passage 2 to 5 . For high-glucose conditions, cells were subjected to 30 $\mathrm{mM}$ D-glucose or $30 \mathrm{mM} \mathrm{L}$-glucose (both MilliporeSigma) serving as osmotic control for a minimum of 24 and a maximum of 5 days. To evaluate effects of V1-cal peptide on 12LOX expression, cells were cultured under high-glucose conditions for 48 hours with or without $1 \mu \mathrm{M}$ V1-cal for the last 12 and 24 hours. One micromolar V1-scr or TAT linker served as control. 12(S)-HpETE was purchased from Enzo Life Sciences, 4-(3-chloro-2-pyridinyl)- $N$-[4-(1,1-dimethylethyl) phenyl]-1-piperazine-carboxamide (BCTC) and baicalein from Tocris, and phenidone from MilliporeSigma. For the assessment of capillary formation, endothelial cells were harvested, and $1.5 \times 10^{4}$ cells were seeded onto extracellular matrix equivalent (Matrigel, BD Biosciences) in triplicate in a 96-well format. Wells were photographed 8 hours later and analyzed using ImageJ software. For immunohistochemistry, HUVECs were grown on glass coverslips, fixed using $4 \%$ paraformaldehyde, and permeabilized with Triton and TRPV1 stained with antiTRPV1 antibody (clone N221/17, UCD/NIH NeuroMab Facility) and anti-Tom20 (sc11415, Santa Cruz Biotechnology).

Cell fractionation and immunohistochemistry. Confluent $10 \mathrm{~cm}$ dishes were used for mitochondria isolation as previously described (50). In brief, endothelial cells were cultured under standard conditions and harvested when confluent using trypsinization. Cell solutions were centrifuged twice 5 minutes at $600 \mathrm{~g}$ followed by different centrifugation steps of the resuspended pellet (in $225 \mathrm{mM}$ mannitol,
$75 \mathrm{mM}$ sucrose, $0.1 \mathrm{mM}$ EGTA, and $30 \mathrm{mM}$ Tris- $\mathrm{HCl}, \mathrm{pH}$ 7.4) for 5 minutes at $600 \mathrm{~g}, 10$ minutes at $7000 \mathrm{~g}, 10$ minutes at $7000 \mathrm{~g}$ (resuspended in $225 \mathrm{mM}$ mannitol, $75 \mathrm{mM}$ sucrose, and $30 \mathrm{mM}$ Tris- $\mathrm{HCl}$, $\mathrm{pH}$ 7.4), and 10 minutes at 10,000 $\mathrm{g}$. Organelle-containing fractions were collected and stored at $-20^{\circ} \mathrm{C}$ in MRB buffer $(250 \mathrm{mM}$ mannitol, 5 mM HEPES, pH 7.4, and 0.5 mM EGTA). Fractions were used for Western blot analysis.

Patch clamp analysis. Patch clamp recordings were made at room temperature from human endothelial cell cultures with an EPC-10 patch clamp amplifier (HEKA Elektronik). Pipettes had a tip resistance of 2-4 M $\Omega$, and series resistance $\left(R_{\mathrm{s}}\right)$ was no more than $15 \mathrm{M} \Omega$. In whole-cell recordings, cells exhibited resting membrane potentials from -10 to $-70 \mathrm{mV}$. After a steady state was established, (E)-capsaicin (Tocris), 12(S)-HpETE (Enzo Life Sciences), 12(S)-HpETE, and BCTC were sequentially applied to the recorded cell using a custom-built perfusion system. In some experiments, cells were loaded with $1 \mu \mathrm{M}$ V1-cal or TAT for 5 minutes before exposure to the above mentioned substances.

Isolation of murine endothelial cells. Murine pulmonary microvascular endothelial cells (MLMVECs) were isolated from 8- to 15-week-old C57BL/6J or Trpv1 ${ }^{-1-}$ mice using magnetic sheep anti-rat IgG-coated Dynabeads (catalog 11035, Invitrogen, Thermo Fisher Scientific) coated with rat anti-mouse CD31 antibody (MEC13.3, BioLegend). In brief, mice were sacrificed by cervical dislocation, and 2-3 lungs were washed in PBS, crushed, and incubated in collagenase solution (20 mg collagenase [C7657, MilliporeSigma], $25 \mu \mathrm{L} 1 \mathrm{M} \mathrm{CaCl}_{2}, 200 \mu \mathrm{L}$ penicillinstreptomycin, $10 \mu \mathrm{L}$ DNase, and $20 \mathrm{~mL}$ PBS) to obtain a single-cell solution. After filtration through a $70 \mu \mathrm{m}$ cell strainer, cell solution was centrifuged 10 minutes at 0.4 relative centrifugal force. Pellet was resuspended in $2 \mathrm{~mL}$ washing medium (DMEM, 10\% FCS, 1\% penicillin-streptomycin) and incubated with CD31-coupled anti-rat IgG-coated Dynabeads on a rotator for 45 minutes at $4^{\circ} \mathrm{C}$. Tubes were placed in a magnetic separator to separate bead-coupled cells that were then plated on gelatin-coated $60 \mathrm{~mm}$ dishes. Upon confluence, sorting was performed twice (passages 2 and 3) with CD31-coupled anti-rat IgG-coated Dynabeads, and obtainment of pure endothelial cells was verified by flow cytometry.

Endothelial cell calcium dynamics. For assessment of cytosolic and mitochondrial calcium changes, endothelial cells were loaded with 1 $\mu \mathrm{M}$ Fluo-4 AM or Rhod-2 AM cell-permeant calcium indicators (Thermo Fisher Scientific) for 30 minutes, washed and equilibrated in fresh medium, harvested, and analyzed using a BD Accuri flow cytometer (BD Biosciences). After 10\% of cell suspensions were used for recording baseline fluorescence intensity, reagents were applied in 10× concentration and fluorescence intensity monitored continuously for up to 5 minutes. 12(S)-HpETE, 15(S)-HpETE (both Enzo Life Sciences), pertussis toxin (MilliporeSigma), capsaicin, V1-cal, V1-scr, and TAT were used for stimulation. Thapsigargin (Tocris) was used to deplete intracellular ER calcium stores at $1 \mu \mathrm{M}$. For calcium-free extracellular conditions, cell suspensions were washed 3 times and medium replaced by calcium-free buffer.

Mitochondrial membrane potential and mitochondrial function. Mitochondrial membrane potential was assessed using the JC-10 mitochondrial membrane potential assay kit (ab112134, Abcam) and a BD Accuri flow cytometer or by life cell imaging after loading of cells with $150 \mathrm{nM}$ tetramethylrhodamine methyl ester (Invitrogen, Thermo Fisher Scientific) or following flow cytometry analysis. In 
vitro mitochondrial function of HUVECs and isolated MLMVECs was investigated using an XFp Seahorse extracellular flux analyzer (Agilent). Endothelial cells were cultured on miniplates until confluence and exposed to either high D- or L-glucose (MilliporeSigma), capsaicin (Tocris), 12(S)-HpETE (Enzo Life Sciences), phenidone (MilliporeSigma), baicalein (Tocris), 15(S)-HpETE, pertussis toxin, EtOH, or DMSO as vehicle control. In some experiments, cells were loaded with $1 \mu \mathrm{M}$ of the peptides V1-cal, V1-scr, and TAT. Media were changed to Seahorse XF DMEM (103575-100, Agilent) before analysis. In some experiments, oligomycin, FCCP, and antimycin/rotenone were used as parts of the Mito Stress Test Kit (103010-100, Agilent).

Western blot. Cell lysates or isolated mitochondria were resolved by SDS-PAGE and transferred to a PVDF membrane. Proteins were detected by anti-TRPV1 (ACC-030, Alomone Labs), anti-12-lipoxygenase (clone OTI1C3, Novus Biologicals), anti- $\beta$-actin (clone BA3R, Thermo Fisher Scientific), anti-vinculin (no. 4650, Cell Signaling Technology), anti-Tom20 (ab56783, Abcam), or anti-GPCR GPR31 antibody (ab75579, Abcam) or membrane fraction cocktail (ab140365, Abcam). For visualization, developing kits for horseradish peroxidase-conjugated secondary antibodies were used.

Study approval. All experimental procedures were approved by the Animal Care and Use Committee of North Rhine-Westphalia, Germany (State Agency for Nature, Environment and Consumer Protection [LANUV]; AZ81-02.04.2018.A059).

Statistics. Statistical analysis was performed using GraphPad Prism 7 software (GraphPad Software Inc.). For comparison of treatment versus control group, Student's 2-tailed $t$ test or, for the comparison of 3 or more groups, 1-way ANOVA followed by Bonferroni's correction for multiple comparisons was used. For repeated comparisons of multiple groups over time, 2-way ANOVA followed by Bonferroni's correction was used. A $P$ value of less than 0.05 was considered statistically significant. Data are presented as mean \pm SEM.

\section{Author contributions}

MO designed research studies, conducted experiments, and acquired and analyzed the data. CB, WL, MM, TS, MK, MND, and JR conducted experiments and acquired and analyzed the data. ERG designed research studies, provided reagents, and revised the manuscript. NMW designed research studies, conducted experiments, acquired and analyzed the data, and wrote the manuscript.

\section{Acknowledgments}

The authors thank Peter Reeh, Institute of Physiology and Pathophysiology, University of Erlangen-Nürnberg, Erlangen, Germany, for providing isolated murine dorsal root ganglia; Jan Siemens for providing Trpv1-knockout mice; and Cornelia Richter-Elsenheimer, Christa Post, Veronica Haase, and Elke Nass for providing excellent technical assistance. This work was funded by grants from the Deutsche Forschungsgemeinschaft (German Research Foundation) to NMW (WA3786/2-1 and WA3786/3-1) and from the NIH to ERG (National Institute of General Medical Sciences GM119522).

Address correspondence to: Nana-Maria Wagner, Department of Anesthesiology, Intensive Care and Pain Medicine, University Hospital Münster, Albert-Schweitzer-Campus 1, 48149 Münster, Germany. Phone: 0049.251.83.46837; Email: nmwagner@uni-muenster.de.

WL's present address is: Department of Anesthesiology, Tongji Hospital, Tongji Medical College, Huazhong University of Science and Technology, Wuhan, China.
1. Patterson CC, et al. Trends and cyclical variation in the incidence of childhood type 1 diabetes in 26 European centres in the 25 year period 1989 2013: a multicentre prospective registration study. Diabetologia. 2019;62(3):408-417.

2. Forbes JM, Cooper ME. Mechanisms of diabetic complications. Physiol Rev. 2013;93(1):137-188.

3. Nascimento AMMAD, Sequeira IJ, Vasconcelos DF, Gandolfi L, Pratesi R, Nóbrega YKM. Endothelial dysfunction in children with type 1 diabetes mellitus. Arch Endocrinol Metab. 2017;61(5):476-483.

4. Kitta Y, et al. Persistent impairment of endothelial vasomotor function has a negative impact on outcome in patients with coronary artery disease. J Am Coll Cardiol. 2009;53(4):323-330.

5. Shenouda SM, et al. Altered mitochondrial dynamics contributes to endothelial dysfunction in diabetes mellitus. Circulation. 2011;124(4):444-453.

6. Lowell BB, Shulman GI. Mitochondrial dysfunction and type 2 diabetes. Science. 2005;307(5708):384-387.

7. Kluge MA, Fetterman JL, Vita JA. Mitochondria and endothelial function. Circ Res. 2013;112(8):1171-1188.

8. Arruda AP, Hotamisligil GS. Calcium homeostasis and organelle function in the pathogenesis of obesity and diabetes. Cell Metab. 2015;22(3):381-397.

9. Patricia MK, et al. Adenoviral delivery of a leu- kocyte-type 12 lipoxygenase ribozyme inhibits effects of glucose and platelet-derived growth factor in vascular endothelial and smooth muscle cells. Circ Res. 2001;88(7):659-665.

10. Hennessy E, et al. Elevated 12-hydroxyeicosatetraenoic acid (12-HETE) levels in serum of individuals with newly diagnosed Type 1 diabetes. Diabet Med. 2017;34(2):292-294.

11. Zhang HJ, et al. 12S-hydroxyeicosatetraenoic acid levels link to coronary artery disease in Type 2 diabetic patients. JEndocrinol Invest. 2013;36(6):385-389.

12. Antonipillai I, Nadler J, Vu EJ, Bughi S, Natarajan R, Horton R. A 12-lipoxygenase product, 12-hydroxyeicosatetraenoic acid, is increased in diabetics with incipient and early renal disease. JClin Endocrinol Metab. 1996;81(5):1940-1945.

13. Hwang SW, et al. Direct activation of capsaicin receptors by products of lipoxygenases: endogenous capsaicin-like substances. Proc Natl Acad Sci U S A. 2000;97(11):6155-6160.

14. Caterina MJ, Schumacher MA, Tominaga M, Rosen TA, Levine JD, Julius D. The capsaicin receptor: a heat-activated ion channel in the pain pathway. Nature. 1997;389(6653):816-824.

15. Earley S, Brayden JE. Transient receptor potential channels in the vasculature. Physiol Rev. 2015;95(2):645-690.

16. Zhao R, Tsang SY. Versatile roles of intracellularly located TRPV1 channel. JCell Physiol.
2017;232(8):1957-1965

17. Hacker K, Medler KF. Mitochondrial calcium buffering contributes to the maintenance of Basal calcium levels in mouse taste cells. JNeurophysiol. 2008;100(4):2177-2191.

18. Stueber T, Eberhardt MJ, Caspi Y, Lev S, Binshtok A, Leffler A. Differential cytotoxicity and intracellular calcium-signalling following activation of the calcium-permeable ion channels TRPV1 and TRPA1. Cell Calcium. 2017;68:34-44.

19. Cole BK, Morris MA, Grzesik WJ, Leone KA, Nadler JL. Adipose tissue-specific deletion of 12/15-lipoxygenase protects mice from the consequences of a high-fat diet. Mediators Inflamm. 2012;2012:851798.

20. Wang Y, et al. Ephrin-B2 controls VEGF-induced angiogenesis and lymphangiogenesis. Nature. 2010;465(7297):483-486.

21. Chen J, et al. Activation of TRPV1 channel by dietary capsaicin improves visceral fat remodeling through connexin43-mediated Ca2+ influx. Cardiovasc Diabetol. 2015;14:22

22. Jahnel R, Dreger M, Gillen C, Bender O, Kurreck J, Hucho F. Biochemical characterization of the vanilloid receptor 1 expressed in a dorsal root ganglia derived cell line. Eur J Biochem. 2001;268(21):5489-5496.

23. Hurt CM, et al. Transient receptor potential vanilloid 1 regulates mitochondrial membrane potential and myocardial reperfusion injury. J Am 
Heart Assoc. 2016;5(9):e003774.

24. Gregg EW, et al. Changes in diabetes-related complications in the United States, 1990-2010. NEnglJ Med. 2014;370(16):1514-1523.

25. Flammer AJ, et al. The assessment of endothelial function: from research into clinical practice. Circulation. 2012;126(6):753-767.

26. Widlansky ME, Hill RB. Mitochondrial regulation of diabetic vascular disease: an emerging opportunity. Transl Res. 2018;202:83-98.

27. Dobrian AD, et al. Role of the 12-lipoxygenase pathway in diabetes pathogenesis and complications. Pharmacol Ther. 2019;195:100-110.

28. Gleissner CA, Galkina E, Nadler JL, Ley K. Mechanisms by which diabetes increases cardiovascular disease. Drug Discov Today Dis Mech. 2007;4(3):131-140.

29. Hammond VJ, O'Donnell VB. Esterified eicosanoids: generation, characterization and function. Biochim Biophys Acta. 2012;1818(10):2403-2412.

30. Tseng MT, Dozier A, Haribabu B, Graham UM. Transendothelial migration of ferric ion in $\mathrm{FeCl}_{3}$ injured murine common carotid artery. Thromb Res. 2006;118(2):275-280.

31. Versari D, Lerman LO, Lerman A. The importance of reendothelialization after arterial injury. Curr Pharm Des. 2007;13(17):1811-1824.

32. Richter C, Frei B, Cerutti PA. Mobilization of mitochondrial $\mathrm{Ca} 2+$ by hydroperoxy-eicosatetraenoic acid. Biochem Biophys Res Commun. 1987;143(2):609-616

33. Napolitano M. The role of the 12(S)-HETE/ GPR31/12-HETER axis in cancer and ischemiareperfusion injury. Biochem Soc Trans. 2019;47(2):743-754.

34. Feinmark SJ, Nardi MA, Harleton E, Hu L, Pan R,
Karpatkin S. The orphan receptor GPR31 is the platelet and HUVEC receptor for 12 (S)-HETE. Blood. 2008;112(11):413.

35. Siangjong L, Gauthier KM, Pfister SL, Smyth EM, Campbell WB. Endothelial 12(S)-HETE vasorelaxation is mediated by thromboxane receptor inhibition in mouse mesenteric arteries. Am J Physiol Heart Circ Physiol. 2013;304(3):H382-H392.

36. Guo $Y$, et al. Identification of the orphan $G$ protein-coupled receptor GPR31 as a receptor for 12-(S)-hydroxyeicosatetraenoic acid. J Biol Chem. 2011;286(39):33832-33840

37. Yang F, et al. Structural mechanism underlying capsaicin binding and activation of the TRPV1 ion channel. Nat Chem Biol. 2015;11(7):518-524.

38. Nita II, et al. Privileged crosstalk between TRPV1 channels and mitochondrial calcium shuttling machinery controls nociception. Biochim Biophys Acta. 2016;1863(12):2868-2880.

39. Arruda AP, Pers BM, Parlakgül G, Güney E, Inouye K, Hotamisligil GS. Chronic enrichment of hepatic endoplasmic reticulum-mitochondria contact leads to mitochondrial dysfunction in obesity. Nat Med. 2014;20(12):1427-1435.

40. Valenzano KJ, et al. N-(4-tertiarybutylphenyl)4-(3-chloropyridin-2-yl)tetrahydropyrazine$1(2 \mathrm{H})$-carbox-amide (BCTC), a novel, orally effective vanilloid receptor 1 antagonist with analgesic properties: I. In vitro characterization and pharmacokinetic properties. J Pharmacol Exp Ther. 2003;306(1):377-386.

41. Bestall SM, et al. Sensory neuronal sensitisation occurs through HMGB-1-RAGE and TRPV1 in high-glucose conditions. J Cell Sci. 2018;131(14):jcs215939.
42. Bishara NB, Ding H. Glucose enhances expression of TRPC1 and calcium entry in endothelial cells. Am J Physiol Heart Circ Physiol. 2010;298(1):H171-H178.

43. Hong S, Wiley JW. Early painful diabetic neuropathy is associated with differential changes in the expression and function of vanilloid receptor 1. J Biol Chem. 2005;280(1):618-627.

44. Randhawa PK, Jaggi AS. A review on potential involvement of $\mathrm{TRPV}_{1}$ channels in ischemiareperfusion injury. J Cardiovasc Pharmacol Ther. 2018;23(1):38-45

45. Wu Y, Gross ER, Qian J. Risks of impaired organ protection with inhibiting transient receptor potential vanilloid 1. Anesthesiology. 2018;129(2):377-378.

46. Taberner FJ, Fernández-Ballester G, FernándezCarvajal A, Ferrer-Montiel A. TRP channels interaction with lipids and its implications in disease. Biochim Biophys Acta. 2015;1848(9):1818-1827.

47. Gavva NR, et al. Molecular determinants of vanilloid sensitivity in TRPV1. J Biol Chem. 2004;279(19):20283-20295.

48. Datar SP, Bhonde RR. Modeling chick to assess diabetes pathogenesis and treatment. Rev Diabet Stud. 2011;8(2):245-253.

49. Liu W, et al. Toll-like receptor 2-deficiency on bone marrow-derived cells augments vascular healing of murine arterial lesions. Life Sci. 2020;242:117189.

50. Wieckowski MR, Giorgi C, Lebiedzinska M, Duszynski J, Pinton P. Isolation of mitochondriaassociated membranes and mitochondria from animal tissues and cells. Nat Protoc. 2009;4(11):1582-1590. 